\title{
MAN. 5 - Agile Method of Qualification for Equipment at Pharmaceutical Industry of Biotechnology: A Case Study at Bio-Manguinhos' Henrique Penna Center
}

Thiago Jorge Teixeira Menezes ${ }^{1 *}$; Carlos Francisco Simões Gomes²; Walter Alexandre dos Santos Junior ${ }^{1}$; Jorge Magalhães ${ }^{3}$; Gabriel Castro Ribeiro Ferreira ${ }^{1}$.

1Fiocruz/Bio-Manguinhos;

2UFF - Universidade Federal Fluminense;

3Fiocruz/Farmanguinhos.

Introduction: The qualification process for equipments isn't still easy to be performed as required by Brazilian Regulatory Agency (Anvisa) with the short time available to start the production. To ensure efficiency and agility during the qualification steps, the work applies an integrated approach at the commissioning stage. A Strategic Management Plan has been developed combining knowledge, practices, procedures and competencies applicable to complex engineering products in order to ensure the safe transfer of the construction and installation phase to the operating phase in a shorter period of time, contributing to higher quality and effectiveness of the final product.

Objective: Apply a strategic plan of agile qualification for use at Bio-Manguinhos 's Henrique Penna Center, assuring the regulatory aspects for production, through robust verifications and standardized steps. Then, to apply the plan and demonstrate the accuracy of the process.

Methodology: A literature review was developed through articles in indexed databases and international guides of a scientific technical structure system. The study and its stages were systematically based on the qualitative and quantitative approaches, with on-site execution of integrated qualification method, which includes the commissioning activities. We generated data that subsidized a gradual construction of a graphing tool for the time spent in this process. Thus, it permeated the comparative analysis between the methodology used and the standard qualification model, without the implemented integration.

Results: Based on the prospective vision of the third industrial revolution and the increasing demand for continuous improvement, this work confirmed, with the new qualification proposal, the guarantee of a reduction in qualification time by about $50 \%$. In this study about 50 equipments were considerd, with impact on the increase of the reliability of the equipments present in the Bio-Manguinhos 's Henrique Penna Center. Reducing efforts and reworks, non-conformities, product quality assurance and equipment compliance are highlighted. Also, it is important to emphasize the acceptability perceived by the Good Manufacturing Practices regulatory agencies for this approach. It is expected that the strategic test Management Plan developed will be used as an orientation guide for new qualification plans, in order to be more effective and faster in the pharmaceutical institutions.

Conclusion: One of the challenges that has been presented to the pharmaceutical industries is the time spent to finish their qualification processes. This requires the integration of committed professionals, towards the constant search for primacy quality. With increasing regulatory requirements associated with short time available and the need for detailed information, many industries have begun to rely on consultancies to assist and execute the qualification approach. Thus, this work identified, demonstrated and applied the qualification process introducing the agile method, ensuring the quality of the final product to be supplied in less time, with greater efficiency and cost reduction.

Keywords: Agile Method; Technological Management; Equipment Qualification 